\title{
Special Issue on Increasing Flexibility in Wireless Software Defined Radio Systems
}

\author{
Sarvpreet Singh ${ }^{1} \cdot$ Marc Adrat $^{1} \cdot$ Gerald Ulbricht $^{2}$
}

Published online: 24 July 2017

(C) Springer Science+Business Media, LLC 2017

After making constant significant advancements in the field of Wireless Software Defined Radios (SDRs), it is now clear that the related technologies are maturing. It is well known that SDRs are inherently flexible by nature. Still, there is a constant push for efforts to keep increasing the flexibility of Wireless Software Defined Radios. This need for increase in flexibility is not just limited to radios in the civil market but also in the tactical market due to increase in coalition forces working together in sensitive areas of the world. Among various ways to increase this flexibility, some of them are using open standards and architectures, improved techniques in digital signal processing and development of more efficient embedded systems. Thus, this Special Issue of the Springer Journal of Signal Processing Systems focuses on the before mentioned areas of advanced SDRs technologies which help in building more efficient and flexible SDRs. This Special Issue is divided into four sections and consisting of nine articles.

The first section explains the role played by the Software Communications Architecture (SCA) in modern SDRs in the tactical domain. The SCA is an open standard developed by the US DoD which is widely accepted because it aims at achieving two major goals of SDRs i.e. interoperability and portability. These features contribute in making tactical SDRs more flexible.

Sarvpreet Singh

sarvpreet.singh@fkie.fraunhofer.de

Marc Adrat

marc.adrat@fkie.fraunhofer.de

Gerald Ulbricht

gerald.ulbricht@iis.fraunhofer.de

1 Fraunhofer FKIE, Fraunhofer Strasse 20, 53343 Wachtberg, Germany

2 Fraunhofer IIS, Am Wolfsmantel 33, 91058 Erlangen, Germany
There are lesson learned from programs which adopted the SCA to help evolve the standard and lay out a vision for the future. Also, proposals are continuously presented to make the standard more flexible by bringing new technologies into the standard.

The second section focuses on Wireless SDRs increasing the flexibility of the latest civil communications standards and technologies like the LTE and 5G. SDR is an enabling technology for spectrum sharing and using SDRs in a cognitive environment is also a research domain which is constantly making advances. Ways of efficiently utilizing the spectrum using Licensed Shared Access (LSA) in an LTE network aims to increase flexibility. Also, managing network resources using artificial intelligence in $5 \mathrm{G}$ networks aims to increase the efficiency of modern civil communication standards.

The third section presents new signal processing techniques and methods to allow a more flexible use of SDRs along different form factors and also to analyze the correlation existing between the computational speed and power consumption in SDRs. This analysis is particularly important to decide on the tradeoffs in developing SDRs for different form factors.

The fourth section aims at highlighting the use of various techniques on embedded systems to optimize the flexibility of the hardware for SDRs. New and advanced frameworks aim to increase the versatility by improving the data transport mechanisms between different processing components. At the same time, using new ideas to change the hardware architecture thereby increasing the dynamic range of SDRs also makes them flexible to use.

\section{Tactical SDRs and the SCA}

The article "A Technical Review of SCA Based Software Defined Radios: Vision, Reality and Current Status" (10.1007/s11265016-1186-6) written by Lee Pucker from Wireless Innovation 
Forum, USA, Dr. Marc Adrat from Fraunhofer FKIE, Germany, Steve Bernier from NordiaSoft, Canada, Dr. Boyd Buchin from Rohde \& Schwarz, Germany, Ken Dingman from Harris Corporation, USA, Dr. Vince Kovarik from VINMA Systems, USA, Jimmie Marks from Raytheon, USA, Raghavan Muralidharan from TataPower SED, India, Sarah Miller from Rockwell Collins, USA, Eric Nicollet from Thales Communications \& Security, France, Mark Turner from Vanguard Wireless Consulting, USA and Alberto Quintana from Indra, Spain presents the evolution and adoption of the SCA standard of the U.S. Department of Defense Joint Tactical Networking Center (JTNC) in collaboration with the Wireless Innovation Forum from version 2.2.2 to version 4.1 in the military communications market and how it is driving a second generation of military SDRs.

The article "Model-Based Systems Engineering: Lessons Learned from the Joint Tactical Radio System" (10.1007/ s11265-016-1218-2) written by Dr. Vincent Joseph Kovarik from VINMA Systems, USA and Raghavan Muralidharan from Tata Power SED, India describes the challenges faced by the Joint Tactical Radio System Program in the USA. The paper puts forth the assertion that a key aspect of the engineering process, i.e. systems engineering was not planned into the program and thus a fundamental cause for many of the issues encountered.

The article "Using OpenCL to Increase SCA Application Portability" (10.1007/s11265-017-1225-y) written by Steve Bernier, François Lévesque, Martin Phisel, Dmitry Zvernik from NordiaSoft, Canada and David Hagood from Aeroflex, USA presents how the Open Computing Language (OpenCL ${ }^{\mathrm{TM}}$ ) can be used together with the Software Communications Architecture to increase the portability of applications that need to perform intensive signal processing. The focus is to increase portability of waveform applications on different processing architectures like the GPPs, DSPs and FPGAs.

\section{Civil Communication Standards}

The article "Field Trial Of Licensed Shared Access With Enhanced Spectrum Controller Power Control Algorithms and LTE Enablers" (10.1007/s11265-016-1170-1) written by Seppo Yrjola, Vesa Hartikainen, Lucia Tudose from Nokia, Finland, Jaakko Ojaniemi from Fairspectrum, Finland, Arto Kivinen from Turku University of Applied Science and Tero Kippola from Centria University of Applied Sciences presents the results from a field trial of the Licensed Shared Access (LSA) concept using TD-LTE network in the 3rd Generation Partnership Project spectrum band $40(2.3-2.4 \mathrm{GHz})$ in Finland. New LSA concept elements, LSA Repository for Incumbent protection information and LSA Controller for controlling the mobile broadband network in the same spectrum band were implemented in the trial environment.

The article "AI based Network and Radio resource management in 5G HetNets" (10.1007/s11265-017-1223-0) written by Giulio Bartoli, Dania Marabissi from University of Florence, Italy and Renato Pucci, Luca Simone Ronga from CNIT, Florence Research Unit, Italy presents ways to reduce unsatisfied user's data rate requests and to reduce energy consumption thus reducing the number of activated cells using an artificial intelligence based approach. It consists of two interdependent decisional cores exchanging information, one aware of physical layer aspects and the other controlling pure network resources.

\section{Advanced Signal Processing}

The article "Design and Analysis of Hierarchically Modulated BICM-ID Receivers With Low Inter-Layer Interferences" (10.1007/s11265-017-1222-1) written by Matthias Tschauner, Md. Farhan Tasnim Oshim, Dr. Marc Adrat, Dr. Markus Antweiler from Fraunhofer FKIE, Germany and Benedikt Eschbach, Prof. Peter Vary from RWTH Aachen, Germany presents a novel methodology to optimize Hierarchically Modulated BitInterleaved Coded Modulation with Iterative Decoding (HM-BICM-ID). The concept permits using radios with varying processing capabilities, e.g. handheld radios, vehicular based radios etc.

The article "Advanced Low Power High Speed Nonlinear Signal Processing: An Analog VLSI Example" (10.1007/ s11265-016-1171-0) written by Giuseppe Oliveri, Dr. Werner G Teich, Prof. Jürgen Lindner, Prof. Hermann Schumacher from Ulm University, Germany and Dr. Mohamad Mostafa from DLR, Germany analyzes the strong link existing between computational speed and power consumption. It demonstrates the potential of signal processing with analog circuits to increase the energy efficiency by some orders of magnitude by choosing channel equalization as an application of nonlinear signal processing with a vector equalizer. It provides an analysis of the equalizer, including the analog circuit design, system-level simulations, and comparisons with the theoretical algorithm.

\section{Embedded Systems}

The article "DFC++ Processing Framework Concept - A Novel Framework Approach for Flexible Signal Processing on Embedded Systems" (10.1007/s11265-016-1174-x) written by Dominik Soller, Thomas Jaumann, Dr. Gerd Kilian from Fraunhofer IIS, Germany and Dr. Jörg Robert, Prof. Albert Heuberger from Friedrich Alexander University, Germany 
proposes the Data Flow Control for $\mathrm{C}++$ processing framework to meet the requirements of increased flexibility and versatility of modern SDRs. It puts focus on the reference pointer based data transport mechanisms responsible for the propagation of user data between different processing components.

The article "Increasing SDR receiver dynamic range by ADC diversity" (10.1007/s11265-017-1250-x) written by Gerald Ulbricht, Jakob Kneißl, Christian Kelm, Dr. Gerd Kilian from Fraunhofer IIS, Germany proposes several techniques to extend the dynamic range for an SDR receiver front-end by stacking multiple ADCs and driving them with different gain factors. It compares the proposed techniques and extends existing analysis tools for the use of arbitrary gain factors. Results are verified by simulations and measurements using an existing low power wide area networks (LPWAN) implementation.

Acknowledgements In this Special Issue of the Springer Journal of Signal Processing Systems, ways to increase the flexibility in the wireless communications systems focusing on the software defined radios are discussed.

As Guest Editors, we express our sincere gratitude and warm thanks to all the reviewers for devoting their time in providing us insightful feedback for this Special Issue. The unconditional support of reviewers for the Editorial Board and their professionalism towards the authors is worth appreciating. We would also like to thank the team at Springer for their work and constant support in setting up this Special Issue.

We hope that this Special Issue will provide valuable insights and widen your understanding on the topic to assist you in your future work.

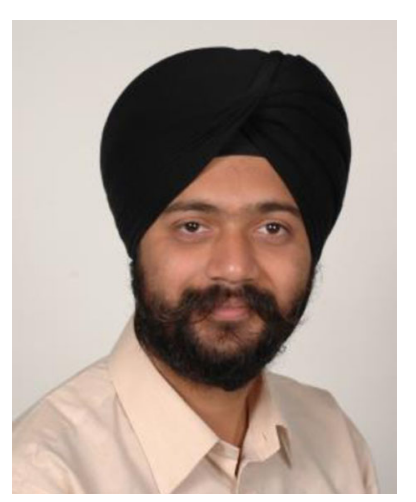

Sarvpreet Singh received his B.E. degree in Computer Science and Engineering from Visvesvaraya Technological University, India in 2003. He received his MSc. degree in Media Informatik from RWTH Aachen University, Germany in 2007. Since July 2007 , he is working as a Research Scientist in the Software Defined Radio Group under the Communications System department of the Fraunhofer Institute for Communication, Information Processing and Ergonomics (FKIE). His current research interests include software defined radio, cognitive radio and waveform development environments focusing on interoperability and portability of waveforms. He has published several technical papers and reports in the above research areas. He has also served as Session Chair, Paper Review Committee Chair and part of Technical Program Committee at several conferences of the Wireless Innovation Forum (WInnF).

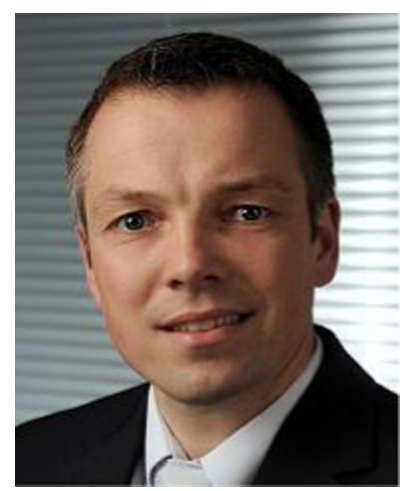

Marc Adrat received his Dipl.Ing. degree in electrical engineering and the Dr.-Ing. degree $(\mathrm{PhD})$ from RWTH Aachen University, Germany, in 1997 and 2003, respectively. Since April 2005, he is with the Fraunhofer Institute for Communication, Information Processing and Ergonomics (FKIE). He is the head of the Software Defined Radio group in the Communication System department. His current research interests include software defined radio, cognitive radio, (military) waveform design as well as concepts for waveform development environments. Matters of particular interest are wideband networking waveforms as well as waveform development environments for portable, interoperable and energy-efficient waveforms. He acts as an Advisor for the WInnF's Coordinating Committee for International SCA Standards.

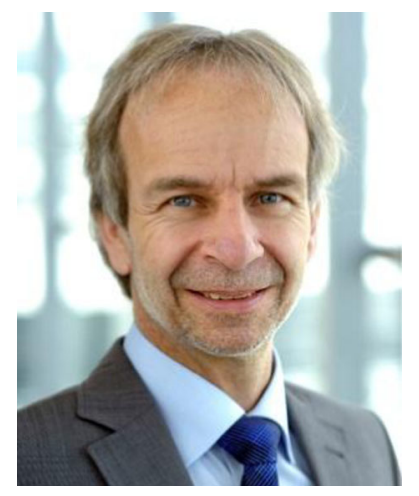

Gerald Ulbricht received his Dipl.-Ing. degree in electrical engineering from the FriedrichAlexander University Erlangen, Germany in October 1990. After University, he joined the Fraunhofer Institute for Integrated Circuits (Fraunhofer IIS) in Erlangen as a Research Scientist designing RF transceiver frontends. Between 1995 and 1998 he worked on CATV wide band amplifier modules at Temic $\mathrm{GmbH}$ and transmitters for GSM base stations at Ericsson Eurolab in Nuremberg. 1998 he returned to the Fraunhofer IIS as a leader of the design group Front-end Technologies within the Radio Communication Systems department. His main research interests are multi-standard RF frontends, Software Defined Radio and the design and linearization of efficient RF power amplifiers. He is holding patents in the context of linear and linearized RF transmitters. Gerald Ulbricht has served as Session Chair and part of Technical Program Committee at several conferences e.g. of the Wireless Innovation Forum (WInnF). 